\title{
Duchenne muscular dystrophy in Wales: a 15 year study, 1971 to 1986
}

\author{
A M NORMAN*, C ROGERS*, J R SIBERT $\dagger$, AND P S HARPER* \\ From *the Institute of Medical Genetics, University of Wales College of Medicine, Heath Park, Cardiff \\ CF4 4XW; and the Department of Child Health, Llandough Hospital, Llandough, South Glamorgan.
}

SUMmARY A register of families with Duchenne muscular dystrophy (DMD) in Wales was set up in 1973 and has been regularly maintained ever since. All women at significant risk in these families were offered estimation of their carrier status by creatine kinase and pedigree analysis. A total of 225 of the 512 women tested was assigned a risk of carrying the DMD gene of less than $5 \%$. One hundred and twenty live births from this group were notified to the register and only one was an affected male. This was the expected number and shows that the risks given were largely accurate. Women given a risk of $5 \%$ or greater were offered fetal sexing with termination of any male. Amniocentesis was of limited value and chorionic villus sampling, introduced in 1984, was acceptable to more women at risk.

The incidence of DMD in Wales dropped from one in 3435 at the beginning of the study to one in 4046 by 1982 , and the proportion of recurrent cases from $40 \%$ to $22 \%$. Common reasons for recurrence were birth of a second affected boy before diagnosis of the first, and failure of families with affected dead members in previous generations to be notified to the register. Maintaining an accurate register of DMD families is an essential tool in their management.

Duchenne muscular dystrophy (DMD) has been the subject of study by one of us (PSH) and his colleagues since late 1971 . Initially the task was to provide genetic counselling for families in south Wales, but subsequently a more general survey and the extension of genetic counselling to north Wales made the collection of data on an all Wales basis feasible. Three other important developments were a study of creatine kinase ranges in obligatory carriers for DMD and in normal controls, ${ }^{1}$ the construction of a regularly maintained genetic register for DMD, and the establishment in Cardiff of a muscular dystrophy clinic allowing genetic counselling to be accompanied by more general support and management, including physiotherapy and occupational therapy. More recently research on localisation of the DMD and Becker muscular dystrophy genes has been a major activity and has been applied to carrier detection, while first trimester chorionic villus sampling has been developed to allow early fetal sexing ${ }^{2}$ and now prenatal diagnosis. Table 1 shows the chronology of these developments.

Here we describe the effect of our activities before the advent of recombinant DNA technology, the application of which is described in the accompanying paper. $^{3}$

\section{Methods}

In our original 1973 study all paediatricians and neurologists in Wales and the adjoining regions were contacted personally and a search was made of

TABLE 1 DMD in Wales, developments 1971 to 1986.

\begin{tabular}{ll}
\hline 1971 & Studies in south Wales families first began. \\
1973 & CK range established for obligatory carriers and normal \\
subjects. & Systematic survey of DMD in Wales; DMD register \\
set up. & Genetic counselling services for DMD extended to \\
north Wales. \\
1976 \\
Cardiff muscular dystrophy clinic started. \\
1979 & First molecular studies in DMD initiated (with \\
1981 & St Mary Hospital, London). \\
1983 & Localisation of DMD gene on X chromosome. \\
1984 & First trimester fetal sexing established. \\
1985 & Systematic DNA analysis undertaken for carrier \\
prediction. & First gene deletions in DMD identified. \\
1986 & First prenatal use of DNA markers. \\
1987 & DMD register placed on microcomputer. \\
\hline
\end{tabular}


TABLE 2 Incidence of DMD in Wales, 1960 to 1984.

\begin{tabular}{lrll}
\hline $\begin{array}{l}\text { Incidence } \\
1960-1973\end{array}$ & & $\begin{array}{l}\text { Incidence } \\
1974-1984\end{array}$ & \\
\hline 1960 & 5 & 1974 & 5 \\
1961 & 11 & 1975 & 3 \\
1962 & 2 & 1976 & 5 \\
1963 & 7 & 1977 & 4 \\
1964 & 7 & 1978 & 5 \\
1965 & 13 & 1979 & 5 \\
1966 & 8 & 1980 & 5 \\
1967 & 2 & 1981 & 4 \\
1968 & 6 & 1982 & 4 \\
1969 & 8 & 1983 & 5 \\
1970 & 3 & 1984 & 5 \\
1971 & 4 & & \\
1972 & 5 & & 50 \\
1973 & 11 & & \\
Total & 92 & & \\
\hline
\end{tabular}

records departments in all general hospitals, following a listing of all diagnoses of muscular dystrophy in the Hospital Activities for Wales. Our subsequent continued activity has shown that this ascertainment was essentially complete for south Wales but not for north Wales, whose specialist medical services are located to a considerable extent outside Wales.

An attempt was made to provide genetic counsel- ling for all female relatives at risk, giving priority to those with prior risk of $5 \%$ or greater of carrying the DMD gene. Since 1974 we have systematically tested all family members at significant risk for carrier status, using our own established normal and carrier ranges for creatine kinase (CK). Children at risk of being carriers have been placed on the DMD register but not usually tested until the age of 15 . All risk estimates have been based on the mean of three separate CK results, integrated with pedigree information and with data from other family members, using Bayesian calculations and the computer program PEDIG. ${ }^{4}$

During the period of the analysis, fetal sexing was the only prenatal procedure available for women at high risk of being a carrier. Before 1984 amniocentesis with termination of any male was offered and subsequently first trimester fetal sexing by chorionic villus sampling became available. All women who underwent these procedures were followed up to assess the outcome. Chorionic villus sampling is the subject of a separate study and data are complete; data on amniocentesis may be less so. All women who were assigned low risk or who opted not to have a prenatal test were asked to notify us of the outcome of any pregnancy. Women in both groups were offered CK analysis on any male at

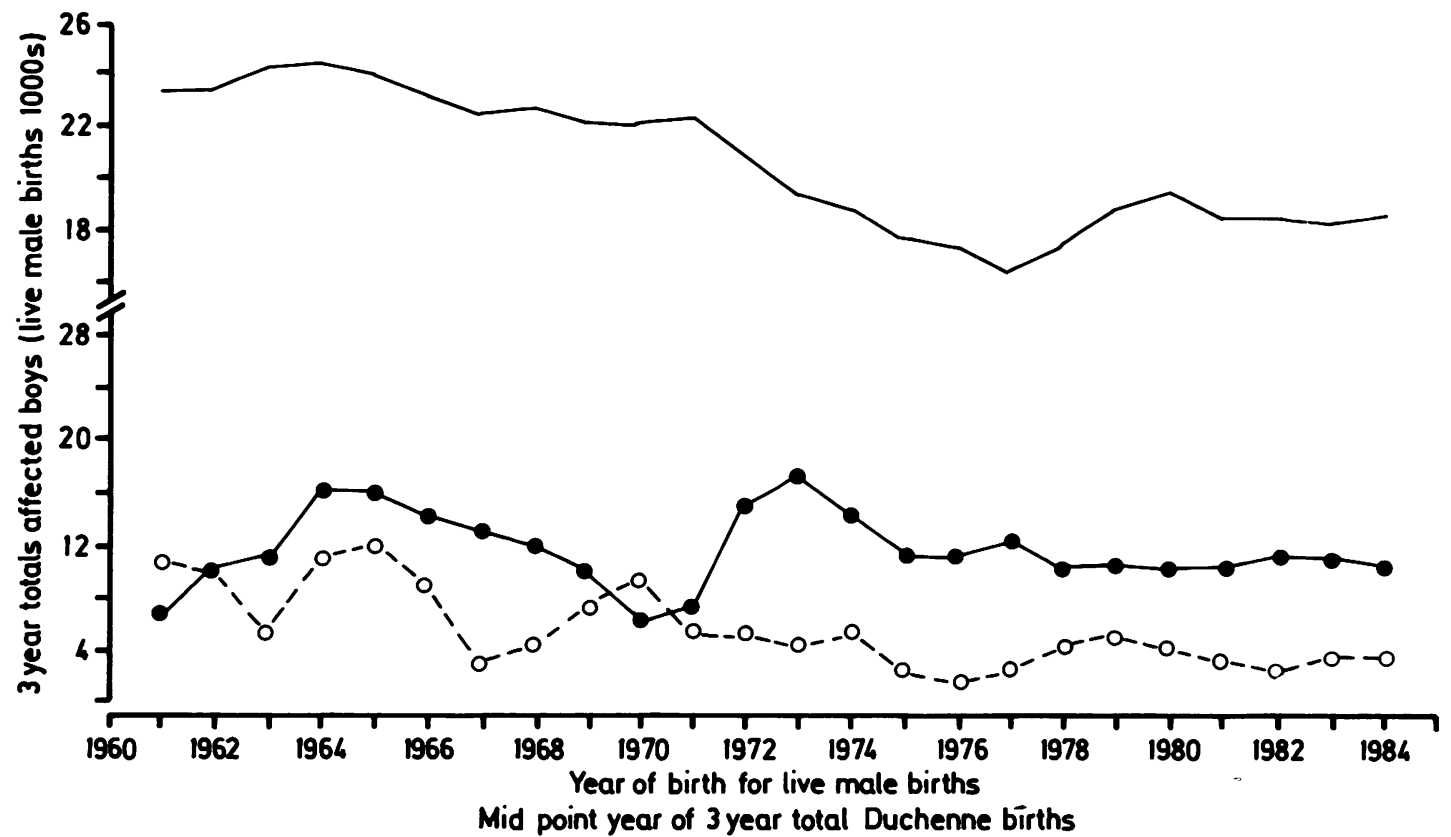

FIG 1 Incidence of Duchenne muscular dystrophy in Wales. Solid line=live male births in Wales. Closed circles= three year totals of isolated DMD births. Open circles=three year totals of recurrent DMD births. 
TABLE 3 Numbers (and percentages) of first and recurrent cases.

\begin{tabular}{llll}
\hline & Total & First cases & Recurrent cases \\
\hline $1960-1973$ & 92 & $57(60 \%)$ & $31(40 \%)$ \\
$1974-1984$ & 50 & $39(78 \%)$ & $10(22 \%)$ \\
\hline
\end{tabular}

TABLE 4 Reasons for recurrent cases, 1974 to 1986.

\begin{tabular}{ll}
\hline Total number of recurrences & 13 \\
$\begin{array}{l}\text { Birth of second affected boy } \\
\text { preceded diagnosis. in first }\end{array}$ & 6 \\
Previous generation affected, but & 4 \\
family not known to the department & 2 \\
High risk pregnancy, prenatal tests declined & 1 \\
Low risk pregnancy, no prenatal test advised & \\
\hline
\end{tabular}

three months of age as a check. Letters were sent to key women in each family in 1986 enquiring about further pregnancies, while 170 such women were interviewed as part of a separate survey in 1987.5 We therefore believe that data on pregnancies are reasonably complete.

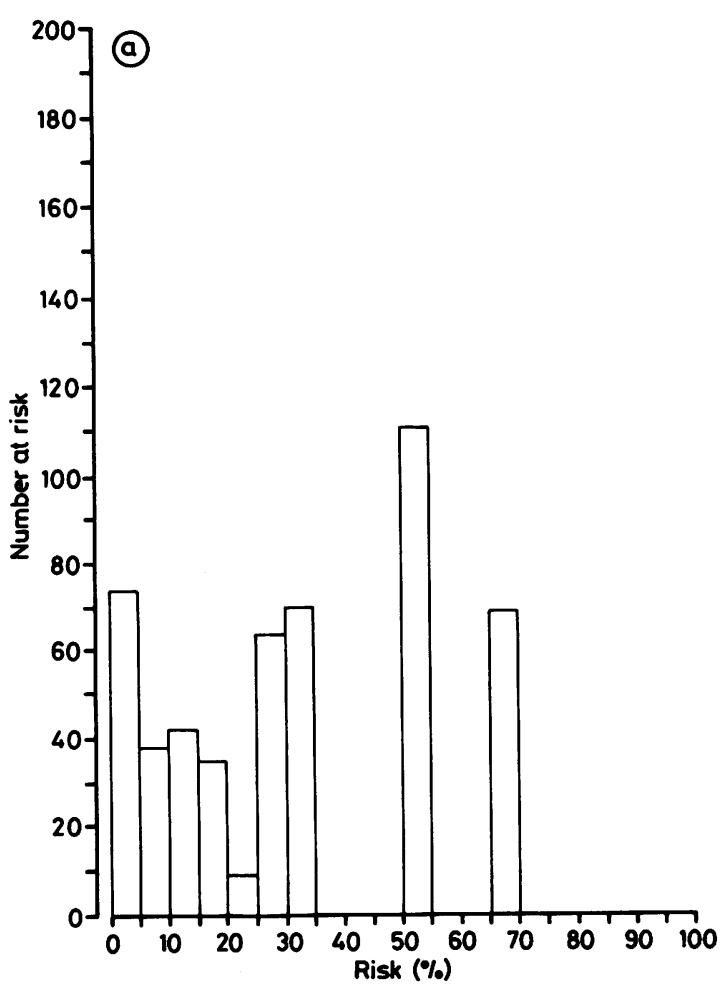

\section{Results}

Table 2 shows the incidence of DMD for the periods 1960 to 1973 and 1974 to 1984 (ascertainment for the later years may still be incomplete). Fig 1 shows the pattern of incidence over the study period taken in three year rolling intervals and divided into first cases in the family and recurrent cases. The annual live male birth rate in Wales over this period is also given. There is no means of preventing the first case of DMD in a kindred, so our strategy must be to prevent subsequent cases by testing the extended family for carrier status. It is thus interesting to assess the incidence of recurrent cases separately, and it is clear from fig 1 that this has diminished, in contrast to the relatively constant level of first cases. The incidence of DMD for 1960 to 1973 was one in 3435 and for 1974 to 1982 one in 4046. This difference was not statistically significant, but suggests prevention of five affected males since the register was set up. Table 3 shows the numbers of first and recurrent cases for the periods 1960 to 1973

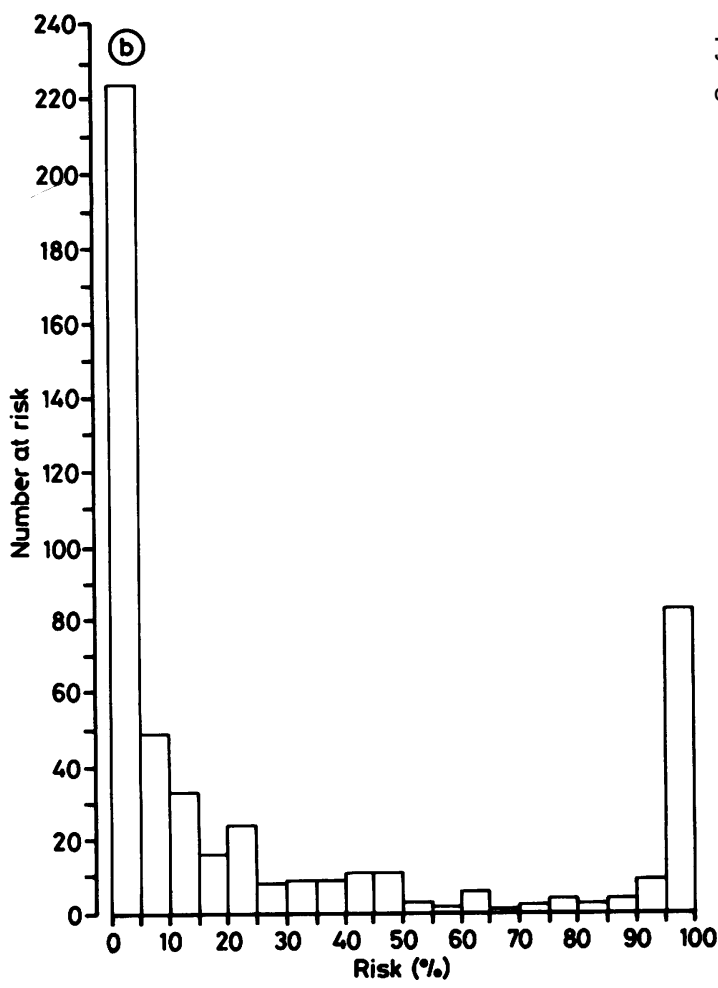

FIG 2 Distribution of carrier risk in 512 women (a) before $C K$ analysis and (b) after CK analysis. 

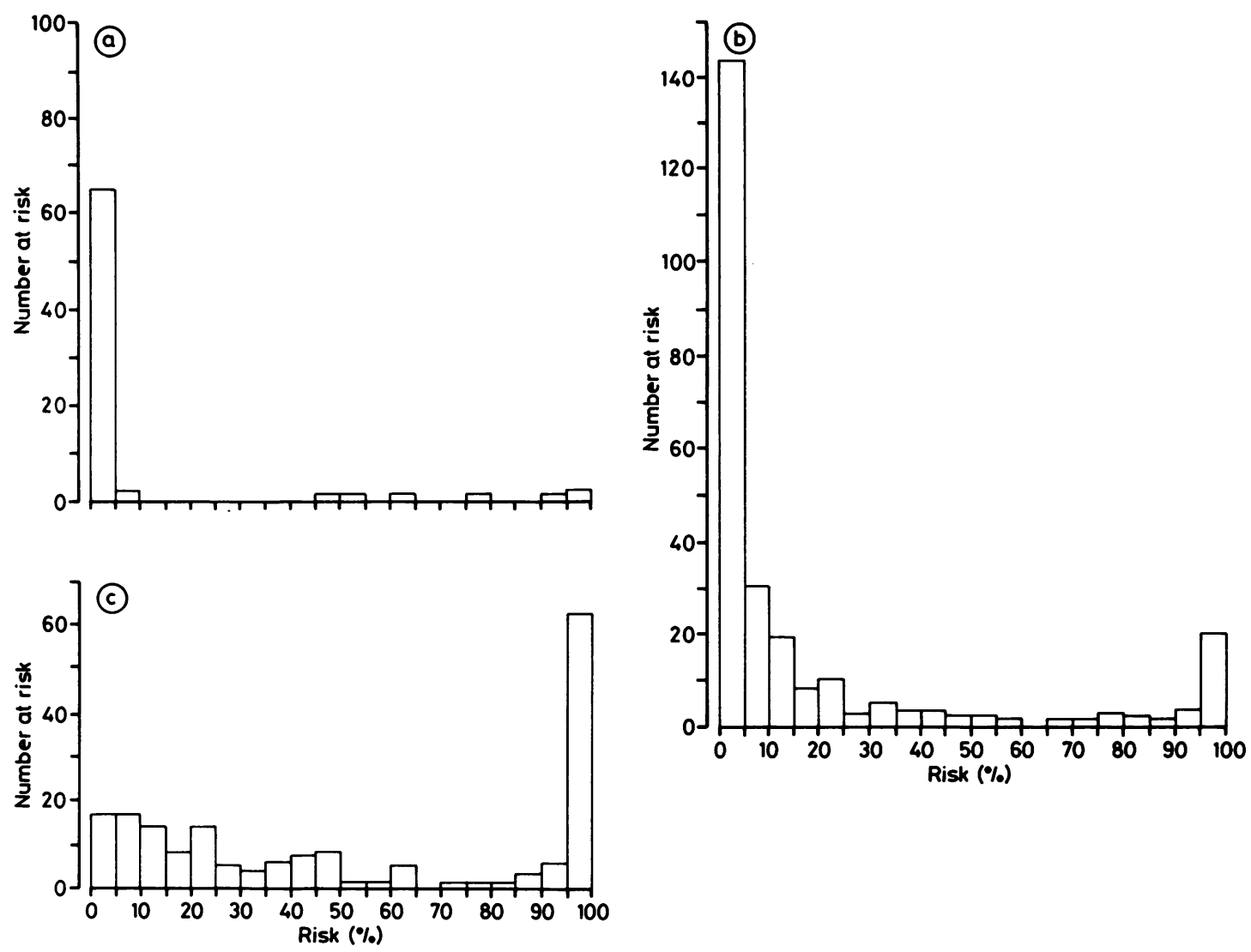

FIG 3 Distribution of carrier risk in 512 women after CK analysis for (a) those at low prior risk (less than 5\% risk), (b) those at intermediate prior risk (5\% or greater, but less than $50 \%$ risk), and (c) those at high prior risk $(50 \%$ or greater risk).

and 1974 to 1984 ; the proportion of recurrent cases is clearly less in the second period. Table 4 shows reasons for recurrent cases during 1974 to 1986.

Fig 2 illustrates how prior risks of carrying the DMD gene based on pedigree analysis were altered by the incorporation of $\mathrm{CK}$ values in 512 women at risk. The number of women at low risk (less than $5 \%$ risk) had increased threefold. Few women remained in the intermediate risk groups. Fig 3 illustrates how the distribution of final risk estimates remains dependent on the prior risk, with most women at medium (risk $5 \%$ or more, but less than $50 \%$ ) or low prior risk being given a low final risk after CK testing.

Table 5 shows the outcome of 245 at risk pregnancies. Taking those women judged to have low risks of being a carrier, 120 pregnancies were recorded between 1973 and 1986, of which 60 were male and only one of these was affected. This suggests that the actual number of gene carriers in this group was one in 30 or $3.3 \%$, a figure consistent with an assignment of individual risk to each woman of less than $5 \%$. It is also clear from table 5 that the uptake of amniocentesis among women at high and medium risk was low. The mean number of pregnancies among high ( $50 \%$ or greater) risk women of reproductive age was 0.35 per woman compared to 0.86 per woman among low risk women, suggesting that refraining from reproduction was the response of many women to receiving a high risk estimate. Nevertheless, further affected males were born to this group. Chorionic villus sampling has been rapidly taken up by women at high risk, and no woman has elected to continue the pregnancy once the fetus was known to be male, in contrast to three women who did so after amniocentesis. 
TABLE 5 Outcome of 245 pregnancies known to the DMD register, 1973 to 1986 . High and medium risk defined as $5 \%$ risk or more, low risk as less than 5\%. Obligate carriers were included in the high prior risk group.

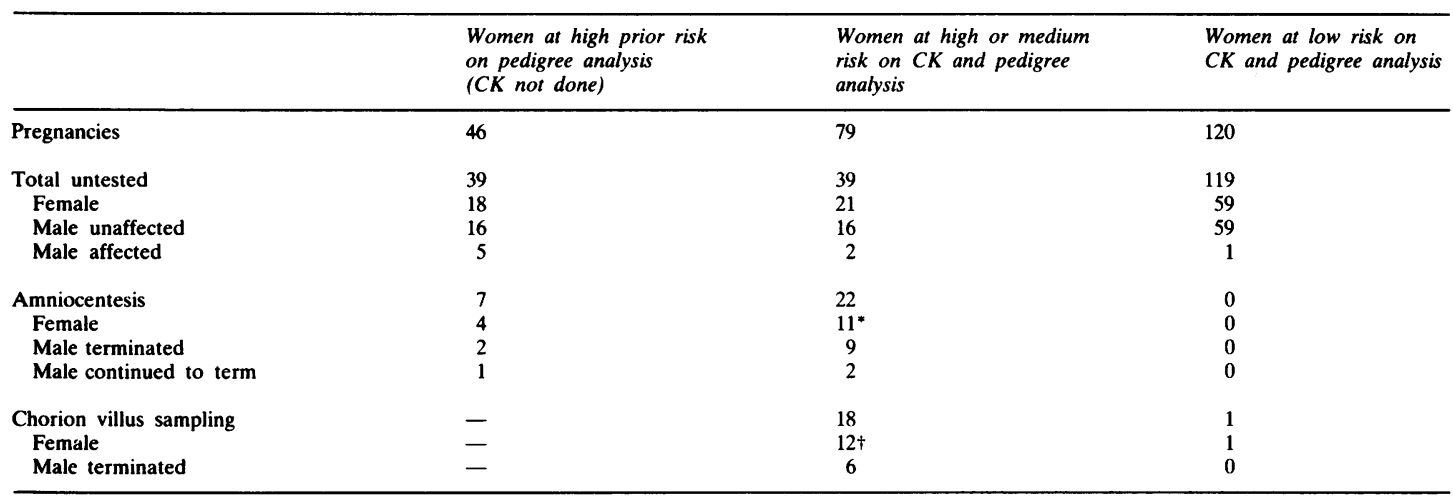

*One female subsequently terminated on other grounds. +Two females subsequently miscarried.

\section{Discussion}

Our experience of setting up and maintaining a genetic register for DMD over 15 years shows that such a register has had some impact on the recurrence of $\mathrm{DMD}$, though there is room for improvement. Creatine kinase analysis has been extremely valuable when used in conjunction with pedigree data. The risks given are largely accurate in carrier detection and do not give false reassurance to the great majority of women tested. Those at low risk are largely able to proceed with pregnancy without prenatal testing. The difficulty lies with those at medium and high risk. Amniocentesis with termination of males in the second trimester has clearly been of limited value. Chorionic villus sampling with first trimester termination of males has been acceptable to more women at risk, so much so that even one woman at $1 \%$ risk elected to undergo it. Nevertheless there are pitfalls, as reported in the accompanying paper.

Since recurrent cases can to some extent be regarded as 'failures' in the prevention of DMD, it is instructive to examine the reasons for them occurring. Table 4 shows that lack of early diagnosis in the first affected boy in the pedigree, and failure of families with a dead affected member in a previous generation to be known to the register, are much more important than 'false' risk assignment by tests. $^{6}$ One response was to institute a creatine kinase screening programme for boys failing to walk at 18 months; this has been reported elsewhere. ${ }^{7}$ It is also evident that all families with an affected boy, or a family history of an affected boy, should be referred for genetic counselling. Although demonstrably not all such families will want or need prenatal diagnosis, they should at least have the opportunity to discuss it.
Our data show that many women at high risk have refrained from, or deliberately delayed, reproduction. Some have done so in the hope that we would eventually be able to offer either prenatal diagnosis or, for those in intermediate risk groups, a significant revision of risk by DNA analysis. An analysis of our attempts to do this follows.

This work has been supported by the Muscular Dystrophy Group of Great Britain and the Muscular Dystrophy Association of America.

\section{References}

1 Sibert JR, Harper PS, Thompson RJ, Newcombe RG. Carrier detection in Duchenne muscular dystrophy. Arch Dis Child 1979:54:534-7.

2 Harper PS, Upadhyaya M, Roberts A, Williams H. Chorionic villus sampling in X-linked genetic disorders. In: Liu DTY, Symonds EM, Golbus MS, eds. Chorionic villus sampling. London: Chapman and Hall, 1987:343-51.

${ }^{3}$ Norman AM, Upadhyaya M, Thomas NST, Roberts K, Harper PS. Duchenne muscular dystrophy in Wales: impact of DNA linkage analysis and cDNA deletion screening. $J$ Med Genet 1989;26:565-71.

${ }^{4}$ Heuch I, Li FHF. PEDIG-a computer programme for calculation of genotype probabilities using phenotype information. Clin Genet 1972;3:501-4.

${ }^{5}$ Norman A, Harper P. A survey of manifesting carriers of Duchenne and Becker muscular dystrophy in Wales. Clin Genet (in press).

6 O'Brien T, Sibert JR, Harper PS. Implications of diagnostic delay in Duchenne muscular dystrophy. $\mathrm{Br}$ Med $J$ 1983;287: $1106-7$.

7 Smith RA, Rogers M, Bradley DM, Sibert JR, Harper PS. Screening for Duchenne muscular dystrophy. Arch Dis Child (in press).

Correspondence to $\mathrm{Dr} \mathrm{A} M$ Norman, Institute of Medical Genetics, University Hospital of Wales, Heath Park, Cardiff CF4 4XN. 